ఠ ORIGINAL RESEARCH

\title{
Abdominal obesity: causal factor or simply a symptom of obesity-related health risk
}

This article was published in the following Dove Press journal:

Diabetes, Metabolic Syndrome and Obesity:Targets and Therapy 3 July 2014

Number of times this article has been viewed

\author{
Sechang $\mathrm{Oh}^{\prime}$ \\ Kiyoji Tanaka ${ }^{2}$ \\ Jin-won $\mathrm{Noh}^{3}$ \\ Rina So ${ }^{2,4}$ \\ Takehiko Tsujimoto 2 \\ Hiroyuki Sasai ${ }^{1,4}$ \\ Mijung Kim ${ }^{5}$ \\ Junichi Shoda' \\ 'Faculty of Medicine, University \\ of Tsukuba, Tsukuba, Ibaraki, Japan; \\ ${ }^{2}$ Faculty of Health and Sports \\ Science, University of Tsukuba, \\ Tsukuba, Ibaraki, Japan; ${ }^{3}$ Department \\ of Healthcare Management, Eulji \\ University, Seongnam-si, Gyeonggi-do, \\ Republic of Korea; ${ }^{4}$ Japan Society \\ for the Promotion of Science, \\ Tokyo, Japan; ${ }^{5}$ Faculty of Life and \\ Environmental Sciences, University \\ of Tsukuba, Tsukuba, Ibaraki, Japan
}

Correspondence: Junichi Shoda Faculty of Medicine, University of Tsukuba, I-I-I Tennodai, Tsukuba, Ibaraki 305-8575, Japan

Tel +8I 298535795

Fax +8I 298535795

Email shodaj@md.tsukuba.ac.jp
Background: Abdominal fat (AF) reduction is advocated in the treatment of obesity-related diseases. Nonetheless, recent studies have shown additional beneficial effects against obesityrelated health risks, independent of AF reduction. Therefore it is important to determine whether AF plays a causal role in promoting metabolic disorders or is simply a symptom of increased obesity-related health risk factors. Clarification of the primary role of AF in the pathogenesis of obesity-related disease is also important.

Objective: This retrospective study was conducted with the objectives of 1) comparison between groups exhibiting equivalent amounts of AF loss that resulted from distinct treatments (exercise and dietary restriction) with respect to degrees of improvement in obesity-related health risk factors and 2) determination of definite differences in the outcomes of obesity-related health risk in subjects receiving identical treatment (exercise) but exhibiting a remarkable difference in AF reduction.

Design: In 66 subjects who completed a 12-week exercise or dietary restriction program, 17 parameters (systolic blood pressure [SBP] and diastolic blood pressure [DBP]; high-sensitivity C-reactive protein [hs-CRP]; leptin, adiponectin, tumor necrosis factor [TNF]- $\alpha$, interleukin [IL]-6; alanine aminotransferase [ALT], gamma glutamyl transpeptidase [ $\gamma \mathrm{GT}]$; lipid profile: high-density lipoprotein cholesterol [HDLC], triglyceride [TG]; fasting plasma glucose [FPG], hemoglobin $\mathrm{A}_{1 \mathrm{c}}\left[\mathrm{HbA}_{1 \mathrm{c}}\right]$, homeostasis model assessment of insulin resistance (HOMA-IR); creatinine, uric acid; and maximal aerobic capacity $\left[\mathrm{VO}_{2} \max \right]$ ) were examined as indicators of obesity-related health risk.

Results: Despite equivalent magnitudes of AF reduction (-29.5\% versus $-30.1 \%)$ in subjects in the exercise and dietary restriction groups (objective 1), ten parameters (SBP, DBP, HDLC, HOMA-IR, uric acid, creatinine, hs-CRP, adiponectin, IL-6, and $\mathrm{VO}_{2} \max$ ) showed significant differences. However, for large AF reduction differences $(-30.1 \%$ versus $-2.8 \%)$ between groups of subjects in the same exercise program (objective 2), only creatinine and $\mathrm{VO}_{2}$ max were different.

Conclusion: It is likely that AF reduction alone is not directly linked to improvement in obesityrelated health risk factors, indicating the need for reexamination of the management for $\mathrm{AF}$ reduction (ie, lifestyle modification) rather than simply targeting reduction of AF.

Keywords: exercise, dietary restriction, intervention study, lifestyle modification, metabolic syndrome

\section{Introduction}

Obesity and being overweight are strongly associated with an increased risk of obesityrelated diseases, including hypertension, diabetes, heart disease, and cancer, in both Western and Asian populations. ${ }^{1,2}$ In particular, an accelerated accumulation of fat in the abdomen, ie, abdominal obesity, has been recognized as a major and causal factor for 
the origin of metabolic disorders prevalent in obesity-related health risks. ${ }^{3,4}$ This is despite the fact that there is still debate regarding the importance of fat distribution, indicating a functional difference between visceral abdominal fat (AF) and subcutaneous AF. ${ }^{5,6}$

Metabolic syndrome has been receiving considerable attention in recent years, and five criteria are recommended for diagnosis, including measurement of waist circumference rather than body mass index (BMI). ${ }^{7,8}$ Furthermore, in Japan, a waist circumference of $\geq 85 \mathrm{~cm}$ is a precondition for diagnosis of metabolic syndrome. ${ }^{9}$ Past research and proposed criteria suggest that abdominal obesity is a prevalent manifestation of and a major factor contributing to obesity-related diseases. Hence, epidemiological and clinical studies have emphasized the necessity of appropriate treatment strategies, including pharmacotherapy and surgical approaches, to reduce the amount of AF. ${ }^{10}$

However, recent findings indicate that lifestyle modifications independent of detectable AF reduction reduce obesityrelated health risk factors. ${ }^{11-14}$ Furthermore, Fabbrini et al ${ }^{15}$ and Dunn et al $^{16}$ obtained specific evidence by omentectomy that existing AF is not the main culprit of metabolic disorders. These findings argue against the commonly held view regarding the causal role of $A F,{ }^{17-19}$ suggesting that the causal link between AF and obesity-related health risk factors might be misinterpreted.

Based on these considerations, we conducted a retrospective analysis to determine whether reduction of AF itself in lifestyle management plays a major or causal role in diminishing obesity-related health risk factors. In other words, we wished to explore whether reduction in AF regardless of therapeutic differences influences the pathophysiology of obesity-related diseases. To answer this question, we first examined whether there was comparable AF reduction following each of two distinct treatment strategies related to lifestyle modification, exercise and dietary restriction, that achieved equivalent beneficial effects on the parameter of obesity-related health risks. Secondly, looking at a marked difference of AF reduction in two groups receiving an identical treatment strategy (regulatory exercise), we evaluated the definite differences between the two groups for a change in the outcomes of obesity-related health risk.

\section{Methods and procedures}

\section{Subjects and classification of groups}

Figure 1 depicts the workflow of enrollment to the program carried out from 2006 to 2010 at the University of Tsukuba

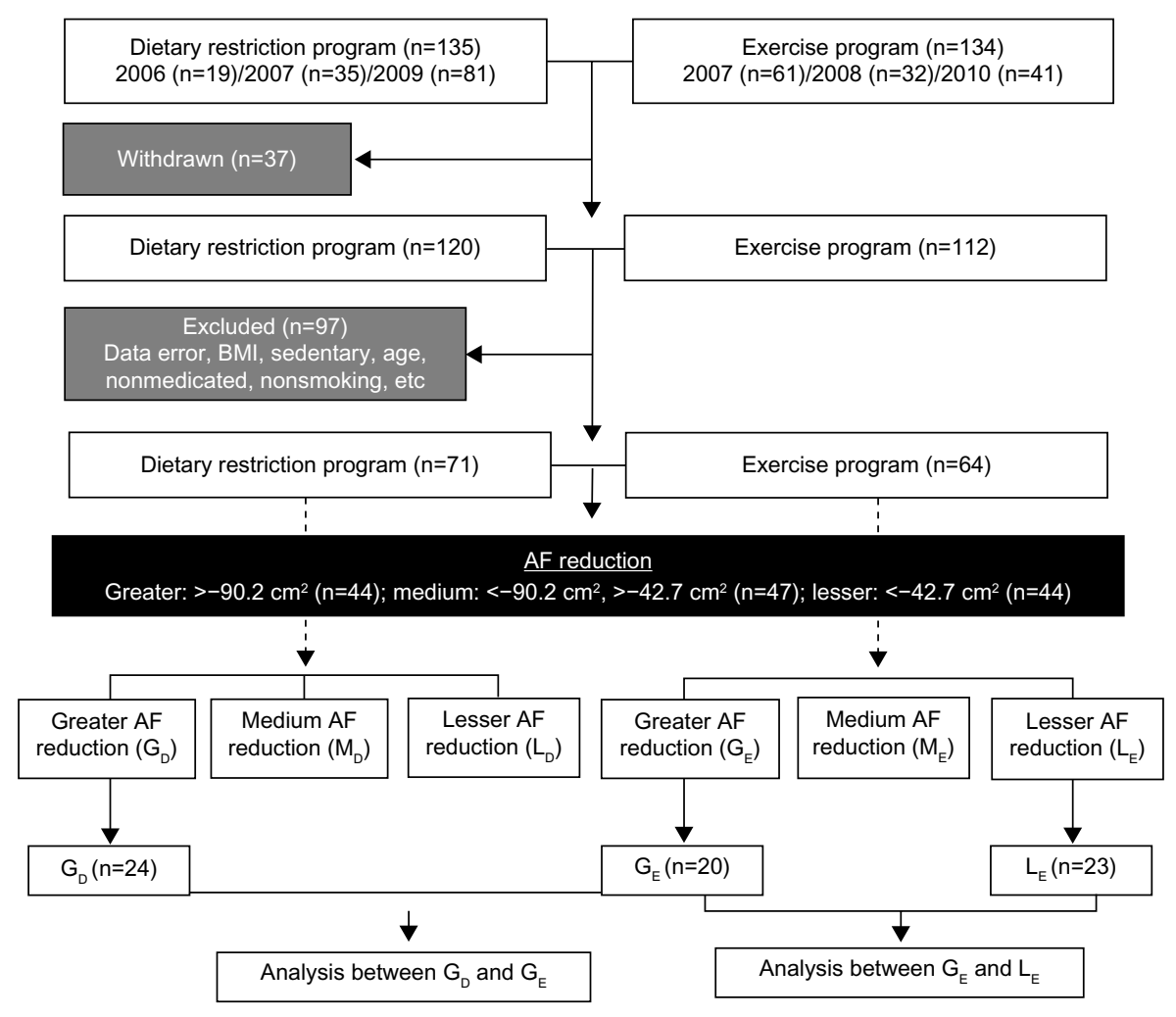

Figure I Flow diagram of the enrollment and classification of study participants.

Notes: $G_{D}=$ group with greater $A F$ reduction, due to dietary restriction; $G_{E}=$ group with greater $A F$ reduction, due to exercise; $L_{D}=$ group with lesser $A F$ reduction, due to dietary restriction; $L_{E}=$ group with lesser $A F$ reduction, due to exercise; $M_{D}=$ group with medium $A F$ reduction, due to dietary restriction; $M_{E}=$ group with medium $A F$ reduction, due to exercise.

Abbreviations: AF, abdominal fat; BMI, body mass index. 
(Tsukuba, Ibaraki, Japan). A total of 269 obese adult men were recruited from Ibaraki Prefecture by advertising a program for obesity reduction via exercise or dietary restriction. Of the 269 initial participants, 232 completed the program. However, of those, 97 participants were found to be ineligible for the study either because of errors or deficits in data collection $(n=15)$ or because they did not satisfy the inclusion criteria $(n=82)$. The inclusion criteria were: sedentary lifestyle (exercise less than $30 \mathrm{~min} /$ day), 35-60 years old, obese (BMI between 25 and $35 \mathrm{~kg} / \mathrm{m}^{2}$ ), attendance $>70 \%$, nonmedicated, and nonsmoking. The 135 participants who satisfied the inclusion criteria were divided into tertiles of the level of total AF area (TAFA) reduction: greater tertile $>-90.2 \mathrm{~cm}^{2}$ (44 men), middle tertile -90.2 to $-42.7 \mathrm{~cm}^{2}$ ( $47 \mathrm{men}$ ), and lesser tertile $<-42.7 \mathrm{~cm}^{2}$ (44 men). At this step, 88 men who were classified as either greater or lesser tertiles were selected. Among those 88 participants, 21 participants belonging to the lesser tertile who participated in the dietary restriction program were excluded from analysis. The remaining 67 participants were further divided according to the completed intervention and were divided in to groups as subjects in the current study: a group with greater AF reduction, due to dietary restriction $\left(G_{D}\right)(n=24)$, a group with greater AF reduction, due to exercise $\left(G_{E}\right)(n=20)$, and a group with lesser AF reduction, due to exercise $\left(\mathrm{L}_{\mathrm{E}}\right)(\mathrm{n}=23)$.

The study protocol was approved by the Institutional Review Board at the University of Tsukuba, Ibaraki, and written informed consent from each participant was obtained.

\section{Dietary restriction regimen}

Subjects in the $\mathrm{G}_{\mathrm{D}}$ group were provided with a dietary restriction of $1,680 \mathrm{kcal}$ per day maintained through a nutritionally balanced diet restriction program consisting of 12 weekly lectures. Subjects kept food diaries; these were monitored by dieticians who later provided recommendations at the weekly lectures. Individual counseling was provided after the classes, to assist the subjects in their adherence to the calorie consumption guidelines. For total daily energy intake assessments, subjects completed a 3-day food record, at baseline and at week 12, under the supervision of a dietician. The food intake records were analyzed using Excel Eiyoukun version 4.0 (Kenpakusya, Tokyo, Japan).

\section{Exercise regimen}

Subjects in the $\mathrm{G}_{\mathrm{E}}$ and $\mathrm{L}_{\mathrm{E}}$ groups participated in an aerobic exercise class for up to 90 minutes/day three times per week for 12 weeks. The exercise program consisted of a 15- to 20-minute warm-up session followed by a 40- to 60 -minute walking and/or jogging session, concluded with a 15- to 20-minute cool-down session. The intensity for exercise was set so that it raised the participants' heart rate to between $60 \%$ and $85 \%$ of their maximum heart rate. Exercise intensities were monitored using a heart rate monitor (Polar RS400'TM; Polar Electro Oy, Kempele, Finland) and an activity monitor with a uniaxial accelerometer (Kenz Lifecorder GS; Suzuken Co, Ltd, Nagoya, Japan). Total daily energy expenditure was assessed using the uniaxial accelerometer every day, from 2 weeks prior to the intervention period and throughout the entire 12-week program.

\section{Abdominal fat area}

The AF area was determined by a computed tomography scan (either an Aquilion ${ }^{\mathrm{TM}} 16$ [Toshiba, Tokyo, Japan] or Somatom ${ }^{\circledR}$ AR.C [Siemens AG, Erlangen, Germany]) in which TAFA, visceral AF area (VAFA), and subcutaneous AF area (SAFA) were measured at the level of the umbilicus. Outcomes were calculated using a computer software program (FatScan; East Japan Institute of Technology Co., Ltd., Osaka, Japan). The intraclass correlations for repeated determinations in our laboratory were 0.99 .

\section{Anthropometry, body composition, and blood pressure}

Body weight was measured using a digital electronic scale (TBF-551; Tanita Corp, Tokyo, Japan). Standing height was measured by a wall-mounted stadiometer (YG-200; Yagami Corp, Nagoya, Japan) and used to calculate BMI $\left(\mathrm{kg} / \mathrm{m}^{2}\right)$. Waist circumference was measured using a fiberglass measuring tape at the level of the umbilicus. Systolic blood pressure (SBP) and diastolic blood pressure (DBP) were measured using a mercury sphygmomanometer (No. 611 Yamasu; Kenzmedico, Saitama, Japan). Segmental body composition, consisting of total fat mass and total lean mass (TLM), was evaluated using dual energy X-ray absorptiometry (either with the Lunar DPX-NT [GE Healthcare, Little Chalfont, UK] or QDR 4500 [Hologic Inc., Bedford, MA, USA]).

\section{Blood analysis}

Blood samples were collected via a butterfly needle inserted into the median cubital vein, following no exercise for 48 hours and a fast status of 12 hours, at baseline and at the 12-week mark. The collected blood was transported on ice to the hospital laboratory, followed by separation of the serum and plasma, and the samples were stored at $-80^{\circ} \mathrm{C}$ until analyzed. Levels of high-density lipoprotein cholesterol 
(HDL-C) and triglyceride (TG) were analyzed enzymatically, fasting plasma glucose (FPG) level was measured by the enzymatic colorimetric method, fasting plasma insulin (FPI) level was measured by a chemiluminescent immunoassay, alanine aminotransferase (ALT) and gamma glutamyl transpeptidase $(\gamma \mathrm{GT})$ levels were measured by the Japan Society of Clinical Chemistry transferable method, hemoglobin $\mathrm{A}_{1 \mathrm{c}}$ $\left(\mathrm{HbA}_{1 \mathrm{c}}\right)$ level was measured by latex agglutination, and high-sensitivity $\mathrm{C}$-reactive protein (hs-CRP) was measured by the fixed time assay method. Commercial enzyme-linked immunosorbent assay (ELISA) kits were used to measure serum levels of total adiponectin (Sekisui Medical Co, Ltd, Tokyo, Japan), tumor necrosis factor alpha (TNF- $\alpha$ ), interleukin 6 (IL-6), and leptin (R\&D Systems Inc., Minneapolis, MN, USA). The intra-assay coefficients of variation for adiponectin, TNF- $\alpha$, IL- 6 , and leptin in our laboratory were $2.2 \%, 4.8 \%, 2.9 \%$, and $3.8 \%$, respectively.

\section{Surrogate markers}

Surrogate markers were used to calculate insulin resistance using the homeostasis model assessment (HOMA-IR), described by Matthews et al. ${ }^{20}$

\section{Statistics}

Descriptive parameters are presented as means \pm standard error. Unpaired Student's $t$-test or Mann-Whitney $U$ test for skewed variables, with the baseline score acting as the clinical parameter, were performed to examine differences between the groups before the program began. In addition, a paired $t$-test, for normally distributed variables, or Wilcoxon's rank sum test, for skewed variables, was used for examining changes as a function of time (at baseline and the 12th week) in each subject. We also compared variables between groups $\left(G_{D}\right.$ and $\left.G_{E}\right)$ that changed from the baseline to the 12 th week, using either analysis of covariance (ANCOVA) or the rank ANCOVA model, with the baseline and fat distribution (VAFA and SAFA) outcomes as a covariate. However, when a comparison was made between the groups $\left(\mathrm{G}_{\mathrm{E}}\right.$ and $\left.\mathrm{L}_{\mathrm{E}}\right)$, we only considered the adjustment for baseline covariates. Statistical analysis was performed using SPSS Statistics for Windows, Version 20.0 (IBM Inc., Armonk, NY, USA). The threshold for significance was set at $P<0.05$.

\section{Results}

\section{Selection of groups and preliminary analysis}

This intervention trial study consisted of 67 subjects divided into three groups, $G_{D}(n=24), G_{E}(n=20)$, and $L_{E}(n=23)$, who satisfied the inclusion criteria as described in the section "Subjects and classification of groups". The selection of the subgroups was based on the results of our preliminary analysis and was done with the aim of clarifying two objectives: objective 1 was a comparison of groups exhibiting an equivalent $\mathrm{AF}$ reduction achieved with different therapeutics, namely, exercise ( $\mathrm{G}_{\mathrm{E}}$ group) and dietary restriction $\left(\mathrm{G}_{\mathrm{D}}\right.$ group); objective 2 examined groups exhibiting a marked AF reduction under identical exercise treatment $\left(\mathrm{G}_{\mathrm{E}}\right.$ and $\mathrm{L}_{\mathrm{E}}$ groups $)$, to understand the outcomes of obesity-related health risk.

For objective 1, we selected the groups in the greater (reduction) tertiles $\left(\mathrm{G}_{\mathrm{D}}\right.$ and $\left.\mathrm{G}_{\mathrm{E}}\right)$ because there were significant differences in independent (VAFA or SAFA) or TAFA reduction between exercise and dietary restriction groups in the medium and lesser tertiles. For objective 2, we selected the groups in the exercise program $\left(\mathrm{G}_{\mathrm{E}}\right.$ and $\left.\mathrm{L}_{\mathrm{E}}\right)$ because we found that among the subjects in the lower tertile of the dietary restriction group, subjects did not incorporate a dietary restriction program into their lifestyle through a food diary and 3-day food record. On the other hand, the subjects in the exercise group $\left(\mathrm{L}_{\mathrm{E}}\right)$ in the lower tertile participated three times a week, with an over $90 \%$ average attendance rate. We also affirmed the results of daily energy expenditure by using a uniaxial accelerometer (data not shown).

\section{Baseline}

A difference in baseline values between groups would have the possibility of influencing the magnitude of changes in outcome in this study. In order to confirm that there were no differences in baseline values between the groups, we first conducted a baseline assessment. The results revealed that there were no significant differences with respect to age $\left(\mathrm{G}_{\mathrm{D}}=47.7\right.$ years, $\mathrm{G}_{\mathrm{E}}=51.8$ years, and $\mathrm{L}_{\mathrm{E}}=48.3$ years $), \mathrm{BMI}$ $\left(\mathrm{G}_{\mathrm{D}}=29.7 \mathrm{~kg} / \mathrm{m}^{2}, \mathrm{G}_{\mathrm{E}}=29.9 \mathrm{~kg} / \mathrm{m}^{2}\right.$, and $\left.\mathrm{L}_{\mathrm{E}}=29.5 \mathrm{~kg} / \mathrm{m}^{2}\right)$, and attendance at classes $\left(\mathrm{G}_{\mathrm{D}}=79.2 \%, \mathrm{G}_{\mathrm{E}}=80.3 \%\right.$, and $\left.\mathrm{L}_{\mathrm{E}}=91.6 \%\right)$ between the groups (data not shown). Furthermore, the means of all parameters were not significantly different between the $\mathrm{G}_{\mathrm{D}}$ and $\mathrm{G}_{\mathrm{E}}$ groups or between in the $\mathrm{G}_{\mathrm{E}}$ and $\mathrm{L}_{\mathrm{E}}$ groups. These results indicated that the baseline values would not interfere with our analysis. Our results for AF area, intervention adherence, anthropometry and body composition, and subsequently, the obesity-related health risk factors are presented in the following sections.

\section{Abdominal fat area}

TAFA, VAFA, and SAFA, the main outcomes for this study, decreased in both the $\mathrm{G}_{\mathrm{D}}$ group $(-29.5 \%,-27.3 \%$, and $-31.6 \%)$ and $\mathrm{G}_{\mathrm{E}}$ group $(-30.1 \%,-29.5 \%$, and $-30.2 \%)$ (Table 1$)$. 


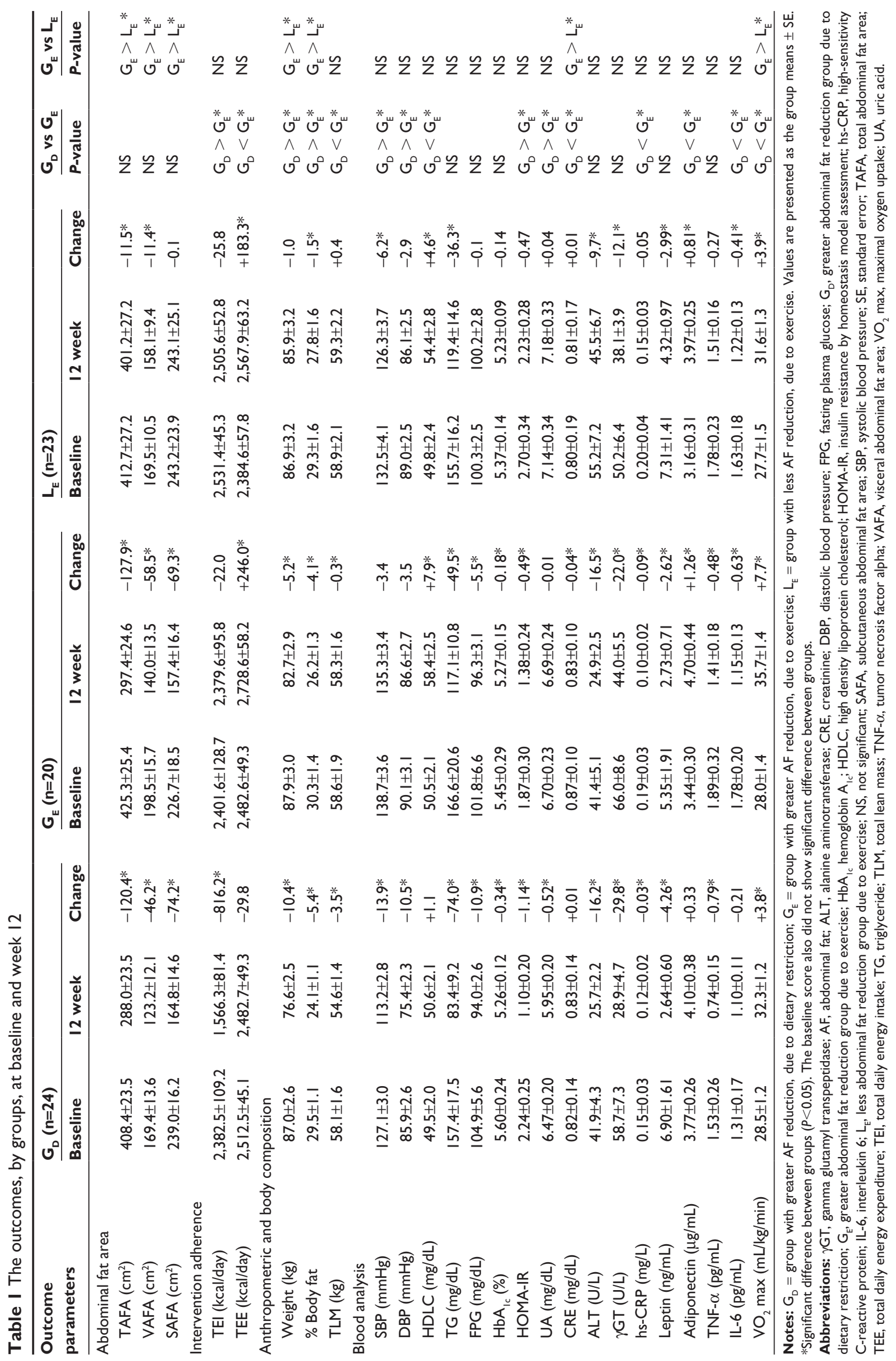


A comparison between the groups showed that there were no significant differences. In the $\mathrm{L}_{\mathrm{E}}$ group, TAFA $(-2.8 \%)$, SAFA (-6.7\%), and VAFA (-0.1\%) significantly decreased at 12 weeks; however, this outcome was significantly lower than that of the $\mathrm{G}_{\mathrm{E}}$ group.

\section{Intervention adherence}

The total daily energy intake in the $\mathrm{G}_{\mathrm{D}}$ group $(-34.3 \%)$ significantly decreased, but there was no significant change in energy intake in either the $\mathrm{G}_{\mathrm{E}}$ group $(-0.9 \%)$ or $\mathrm{L}_{\mathrm{E}}$ group $(-1.0 \%)$ (Table 1$)$. Total daily energy expenditure, assessed using a uniaxial accelerometer, significantly increased in the $\mathrm{G}_{\mathrm{E}}$ group (+10.9\%) and $\mathrm{L}_{\mathrm{E}}$ group $(+7.7 \%)$; however, there was no significant change in the $\mathrm{G}_{\mathrm{D}}$ group $(-1.2 \%)$. When an intergroup comparison was made, significant differences were found in the total daily energy expenditure and total daily energy intake between the $\mathrm{G}_{\mathrm{D}}$ and $\mathrm{G}_{\mathrm{E}}$ groups but not between the $\mathrm{G}_{\mathrm{E}}$ and $\mathrm{L}_{\mathrm{E}}$ groups.

\section{Anthropometry and body composition}

In the $\mathrm{G}_{\mathrm{D}}$ group, the entire outcome (weight, $-12.0 \%$; $\%$ body fat, $-18.3 \%$ ) including TLM (-6.0\%) significantly decreased (Table 1$)$. In the $\mathrm{G}_{\mathrm{E}}$ group, TLM ( $\left.-0.5 \%\right)$ showed no significant change after the 12 -week intervention, but weight $(-5.9 \%)$ and $\%$ body fat $(-13.5 \%)$ showed significant differences. A comparison between the groups showed that the three outcomes, except TLM, in anthropometry and body composition in the $G_{D}$ group were significantly improved compared with those in the $\mathrm{G}_{\mathrm{E}}$ group. In the $\mathrm{L}_{\mathrm{E}}$ group, weight $(-1.2 \%), \%$ body fat $(-5.2 \%)$, and TLM $(+0.7 \%)$ were not significantly changed, but body fat percentage significantly improved from baseline to the 12 th week. When an intergroup comparison was made, significant differences were found for all outcomes except TLM $(P=0.18)$, between the $\mathrm{G}_{\mathrm{E}}$ and $\mathrm{L}_{\mathrm{E}}$ groups.

\section{Obesity-related health risk factors}

Among the 17 parameters of obesity-related health risks, 13 parameters in the $\mathrm{G}_{\mathrm{D}}$ group (SBP $[-10.9 \%$, DBP [-12.3\%], TG [-47.0\%], FPG [-10.4\%], $\mathrm{HbA}_{1 \mathrm{c}}$ [-6.1\%], HOMA-IR [-50.9\%], uric acid [UA] [-7.8\%],

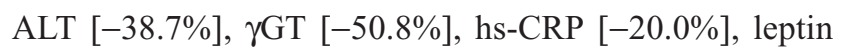
[-87.1\%], TNF- $\alpha[-51.6 \%]$, and $\left.\mathrm{VO}_{2} \max [+13.3 \%]\right), 13$ parameters in the $\mathrm{G}_{\mathrm{E}}$ group (HDL-C [+8.9\%], TG $[-29.7 \%]$, FPG [-5.4\%], $\mathrm{HbA}_{1 \mathrm{c}}[-3.3 \%]$, HOMA-IR [-26.2\%], creati-

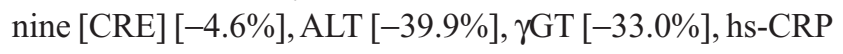
$[-47.4 \%]$, leptin $[-49.0 \%]$, adiponectin [+36.3\%], IL-6 $[-35.4 \%]$, and $\left.\mathrm{VO}_{2} \max [+27.5 \%]\right)$, and nine parameters in the $\mathrm{L}_{\mathrm{E}}$ group (SBP [-4.7\%], HDL-C [+9.2\%], TG [-23.3\%],

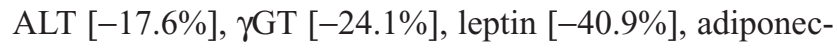
tin $[+25.3 \%]$, IL-6 [-25.2\%], and $\left.\mathrm{VO}_{2} \max [+14.1 \%]\right)$ improved significantly during the 12-week program (Table 1). Parameters that did not improve in the $\mathrm{G}_{\mathrm{D}}$ group were HDL-C $(+2.2 \%)$, CRE (+1.2\%), IL-6 (-16.0\%), and adiponectin $(+8.8 \%)$. Parameters that did not improve in the $\mathrm{G}_{\mathrm{E}}$ group were SBP (-2.5\%), DBP (-3.8\%), UA (-0.2\%), and TNF- $\alpha$ $(-25.4 \%)$, and parameters that did not improve in the $\mathrm{L}_{\mathrm{E}}$ group were DBP $(-3.3 \%)$, FPG $(+0.1 \%), \mathrm{HbA}_{1 \mathrm{c}}(-2.6 \%)$, HOMA-IR (-17.4\%), UA (+0.6\%), CRE (+1.3\%), hs-CRP $(-25.0 \%)$, and TNF- $\alpha(-15.2 \%)$. Interestingly and despite equivalent levels of (VAFA or SAFA) or TAFA reduction in the $G_{D}$ and $G_{E}$ groups, among the 17 parameters, ten outcomes exhibited significant differences between $G_{D}$ and $\mathrm{G}_{\mathrm{E}}$ groups (greater improvements were seen in $\mathrm{G}_{\mathrm{E}}$ than $\mathrm{G}_{\mathrm{D}}$, in HDLC, CRE, hs-CRP, adiponectin, IL-6, and $\mathrm{VO}_{2}$ max; greater improvements were seen in $G_{D}$ than $G_{E}$, in $S B P$, DBP, HOMA-IR, and UA). The six parameters not showing differences were TG, FPG, $\mathrm{HbA}_{1 \mathrm{c}}$, ALT, $\gamma \mathrm{GT}$, leptin, and TNF- $\alpha$. In addition, even though there was a substantial difference in the amount of AF reduction between the $G_{E}$ and $\mathrm{L}_{\mathrm{E}}$ groups, 15 parameters showed no significant differences in these two groups. The two parameters with significant differences were CRE and $\mathrm{VO}_{2}$ max.

\section{Discussion}

Our retrospective study design and selection criteria focused on $\mathrm{AF}$ reduction, with the aim of obtaining new evidence for the need to reevaluate the link between AF and obesity-related health risk factors. The major finding of this study was that equivalent reductions in TAFA, including VAFA and SAFA, achieved by distinct treatment strategies, ie, exercise and dietary restriction, did not result in comparable reduction in the degree of risk factors. In addition, with respect to the remarkable differences in AF reduction between the two groups using an identical treatment (exercise), no definite difference in the risk factors was seen. Our data indicate that the effects of exercise are independent of detectable AF reduction. To the best of our knowledge, our data provide the first evidence from a lifestyle management-related study that beneficial effects against increased risk factors differ according to the therapeutic attempt, regardless of AF reduction.

Most obesity-related studies have shown that the role of visceral $\mathrm{AF}$ is more crucial than that of subcutaneous $\mathrm{AF}$ for obesity-related health risk factors. ${ }^{4,21,22}$ However, our findings do not support those observations. In fact, a causeand-effect relationship between visceral AF and metabolic 
disorders has yet to be demonstrated in humans, ${ }^{18}$ and the importance and primary role of fat distribution are still being debated. ${ }^{5,6}$ From the standpoint of supporting the importance of visceral AF, two main mechanisms have been reported: 1) portal drainage of excessive nonesterified fatty acids (NEFAs) and 2) upregulation of inflammatory cytokines from visceral $\mathrm{AF}^{4}$ However, these mechanisms are debatable in the sense that there are portal and systemic circulations of most of the NEFAs, and proinflammatory cytokines originate from subcutaneous AF, not from visceral AF. ${ }^{6,23}$ Also, NEFAs and proinflammatory cytokines from visceral AF escape metabolism by the liver and enter systemic circulation to contribute to lipotoxicity in other internal organs. ${ }^{24}$ Recently, Fabbrini et a ${ }^{15}$ demonstrated these contradictions by reporting that visceral AF removal by omentectomy did not improve glucose effectiveness, $\beta$-cell function, or metabolic disorders, including insulin sensitivity. Thus, the question arises, "Is subcutaneous AF more important?" Subcutaneous AF might have relatively little contribution to obesity-related health risk factors since, according to Klein et $\mathrm{al}^{25}$ and Dunn et al, ${ }^{16}$ abdominal liposuction did not improve metabolic disorders, including insulin resistance, inflammation, and other risks. These previous findings are consistent with our results showing that AF, either as VAFA or SAFA or as TAFA, was not an important cause of obesity-related health risk factors. The above observation also indicated that the mechanism responsible for the relationship between $\mathrm{AF}$ and metabolic disorders is ambiguous, and AF loss alone does not have metabolic benefits.

In the present study, the $\mathrm{L}_{\mathrm{E}}$ group showed clear improvements for most obesity-related health risk factors without detectable AF reduction after 12 weeks. From these results, we further confirmed the beneficial effect of exercise in the prevention and treatment of metabolic disorders. In this context, it should be noted that serum adiponectin levels, with increasing physical ability, were markedly enhanced by the exercise program. Adiponectin, which improves insulin resistance and enhances the burning of fatty acids, has antiinflammatory and insulin resistance effects; ${ }^{26,27}$ according to the results obtained in this study, it is likely that an increased level of adiponectin has a link with the improved levels of the parameters of metabolic disorders related to dyslipidemia, insulin resistance, hyperglycemia, inflammation, and liver function. Previous studies have also shown that increased exercise improves the levels of adenosine monophosphate (AMP)-activated protein kinase ${ }^{28}$ lipoprotein lipase ${ }^{29}$ retinolbinding protein $4,{ }^{13}$ nuclear factor-E2-related factor- $2,{ }^{30}$ and myokines. ${ }^{31}$ These physiological activations of molecular factors through exercise, regardless of AF reduction, might be the key point for improvement in the $\mathrm{L}_{\mathrm{E}}$ group.

In conclusion, although most clinical and animal studies have warned about the risks of AF, some previous findings (based on surgical methods) and the results of the present study suggest that reduction of AF, either as VAFA or SAFA or as TAFA, alone is not likely to be directly linked to improvement in obesity-related health risk factors. Despite these data and conclusions made, we have not yet determined a specific mechanism for the role of AF. Nevertheless, our results indicate a new direction, for further investigating and clarifying the importance of AF reduction management via lifestyle modification.

\section{Acknowledgments}

We are grateful to Professor Randeep Rakwal (Organization for Educational Initiatives) of the University of Tsukuba for critical review of the manuscript and constructive comments. This work was supported in part by Grants-in-Aid for Scientific Research, from the Ministry of Education, Culture, Sports, Science and Technology, Japan (numbers 24390488 , 25282212, 25282172, and 25293278).

\section{Disclosure}

The authors report no conflicts of interest in this work.

\section{References}

1. Boffetta P, McLerran D, Chen Y, et al. Body mass index and diabetes in Asia: a cross-sectional pooled analysis of 900,000 individuals in the Asia cohort consortium. PLoS One. 2011;6(6):e19930.

2. Poirier P, Giles TD, Bray GA, et al. Obesity and cardiovascular disease: pathophysiology, evaluation, and effect of weight loss. Arterioscler Thromb Vasc Biol. 2006;26(5):968-976.

3. Ritchie SA, Connell JM. The link between abdominal obesity, metabolic syndrome and cardiovascular disease. Nutr Metab Cardiovasc Dis. 2007; 17(4):319-326

4. Després JP, Lemieux I, Bergeron J, et al. Abdominal obesity and the metabolic syndrome: contribution to global cardiometabolic risk. Arterioscler Thromb Vasc Biol. 2008;28(6):1039-1049.

5. Goodpaster BH, Thaete FL, Simoneau JA, Kelley DE. Subcutaneous abdominal fat and thigh muscle composition predict insulin sensitivity independently of visceral fat. Diabetes. 1997;46(10): 1579-1585.

6. Klein S. The case of visceral fat: argument for the defense. J Clin Invest. 2004;113(11):1530-1532.

7. Alberti KG, Zimmet P, Shaw J; IDF Epidemiology Task Force Consensus Group. The metabolic syndrome - a new worldwide definition. Lancet. 2005;366(9491):1059-1062.

8. Expert Panel on Detection, Evaluation, and Treatment of High Blood Cholesterol in Adults. Executive Summary of The Third Report of The National Cholesterol Education Program (NCEP) Expert Panel on Detection, Evaluation, And Treatment of High Blood Cholesterol In Adults (Adult Treatment Panel III). JAMA. 2001;285(19): 2486-2497.

9. Matsuzawa Y. Metabolic syndrome - definition and diagnostic criteria in Japan. J Atheroscler Thromb. 2005;12(6):301. 
10. Coutinho T, Goel K, Corrêa de Sá D, et al. Central obesity and survival in subjects with coronary artery disease: a systematic review of the literature and collaborative analysis with individual subject data. J Am Coll Cardiol. 2011;57(19):1877-1886.

11. Kantartzis K, Thamer C, Peter A, et al. High cardiorespiratory fitness is an independent predictor of the reduction in liver fat during a lifestyle intervention in non-alcoholic fatty liver disease. Gut. 2009;58(9): 1281-1288.

12. Esmaillzadeh A, Kimiagar M, Mehrabi Y, Azadbakht L, Hu FB, Willett WC. Fruit and vegetable intakes, C-reactive protein, and the metabolic syndrome. Am J Clin Nutr. 2006;84(6):1489-1497.

13. Lim S, Choi SH, Jeong IK, et al. Insulin-sensitizing effects of exercise on adiponectin and retinol-binding protein-4 concentrations in young and middle-aged women. J Clin Endocrinol Metab. 2008;93(6): 2263-2268.

14. Eguchi E, Iso H, Tanabe N, et al. Japan Collaborative Cohort Study Group. Healthy lifestyle behaviours and cardiovascular mortality among Japanese men and women: the Japan collaborative cohort study. Eur Heart J. 2012;33(4):467-477.

15. Fabbrini E, Tamboli RA, Magkos F, et al. Surgical removal of omental fat does not improve insulin sensitivity and cardiovascular risk factors in obese adults. Gastroenterology. 2010;139(2):448-455.

16. Dunn JP, Abumrad NN, Breitman I, et al. Hepatic and peripheral insulin sensitivity and diabetes remission at 1 month after Roux-en-Y gastric bypass surgery in patients randomized to omentectomy. Diabetes Care. 2012;35(1):137-142.

17. Nakanishi-Minami T, Kishida K, Nakagawa Y, et al. Carotid intima-media thickness, but not visceral fat area or adiponectin, correlates with intracoronary stenosis detected by multislice computed tomography in people with type 2 diabetes and hypertension. Diabetes Res Clin Pract. 2012;95(1):e23-e26.

18. Fabbrini E, Magkos F, Mohammed BS, et al. Intrahepatic fat, not visceral fat, is linked with metabolic complications of obesity. Proc Natl Acad Sci U S A. 2009;106(36):15430-15435.

19. Chu MP, Klopfenstein BJ, Krisky CM, et al. Intrahepatic lipid, not visceral or muscle fat, is correlated with insulin resistance in older, female rhesus macaques. Obesity (Silver Spring). 2013;21(10):2021-2028.
20. Matthews DR, Hosker JP, Rudenski AS, Naylor BA, Treacher DF, Turner RC. Homeostasis model assessment: insulin resistance and beta-cell function from fasting plasma glucose and insulin concentrations in man. Diabetologia. 1985;28(7):412-419.

21. Item F, Konrad D. Visceral fat and metabolic inflammation: the portal theory revisited. Obes Rev. 2012;13 Supp1 2:S30-S39.

22. Bruun JM, Lihn AS, Pedersen SB, Richelsen B. Monocyte chemoattractant protein-1 release is higher in visceral than subcutaneous human adipose tissue (AT): implication of macrophages resident in the AT. J Clin Endocrinol Metab. 2005;90(4):2282-2289.

23. Basso LV, Havel RJ. Hepatic metabolism of free fatty acids in normal and diabetic dogs. J Clin Invest. 1970;49(3):537-547.

24. Jensen MD. Is visceral fat involved in the pathogenesis of the metabolic syndrome? Human model. Obesity (Silver Spring). 2006; 14 Suppl 1:20S-24S.

25. Klein S, Fontana L, Young VL, et al. Absence of an effect of liposuction on insulin action and risk factors for coronary heart disease. $N$ Engl J Med. 2004;350(25):2549-2557.

26. Ahima RS. Metabolic actions of adipocyte hormones: focus on adiponectin. Obesity (Silver Spring). 2006;14 Suppl 1:9S-15S.

27. Xu A, Wang Y, Keshaw H, Xu LY, Lam KS, Cooper GJ. The fat-derived hormone adiponectin alleviates alcoholic and nonalcoholic fatty liver diseases in mice. J Clin Invest. 2003;112(1):91-100.

28. Richter EA, Ruderman NB. AMPK and the biochemistry of exercise: implications for human health and disease. Biochem J. 2009;418(2):261-275.

29. Miyashita M, Eto M, Sasai H, Tsujimoto T, Nomata Y, Tanaka K. Twelve-week jogging training increases pre-heparin serum lipoprotein lipase concentrations in overweight/obese middle-aged men. J Atheroscler Thromb. 2010;17(1):21-29.

30. Kim SY, Lee YS, Surh YJ. Effects of exercise on inflammatory and antioxidative responses in human peripheral blood mononuclear cells: NF-kB and Nrf2 as potential mediators. Free Radic. Biol. Med. 2011;51(Suppl):S13-S14.

31. Brandt C, Pedersen BK. The role of exercise-induced myokines in muscle homeostasis and the defense against chronic diseases. J Biomed Biotechnol. 2010;2010:520258.

\section{Publish your work in this journal}

Diabetes, Metabolic Syndrome and Obesity: Targets and Therapy is an international, peer-reviewed open-access journal committed to the rapid publication of the latest laboratory and clinical findings in the fields of diabetes, metabolic syndrome and obesity research. Original research, review, case reports, hypothesis formation, expert opinion and commentaries are all considered for publication. The manuscript management system is completely online and includes a very quick and fair peer-review system, which is all easy to use. Visit http://www.dovepress.com/testimonials.php to read real quotes from published authors. 